\title{
Threshold Resummation and the Total Cross Section for Top Quark Production $^{1}$
}

\author{
Edmond L. Berger and Harry Contopanagos \\ High Energy Physics Division \\ Argonne National Laboratory, Argonne, Illinois 60439
}

\begin{abstract}
We discuss the motivation for resummation of the effects of initialstate soft gluon radiation, to all orders in the strong coupling strength, for processes in which the near-threshold region in the partonic subenergy is important. We summarize our calculation of the total cross section for top quark production at hadron colliders. Comments are included on the differences between our treatment of subleading logarithmic terms and other methods.
\end{abstract}

\section{INTRODUCTION AND MOTIVATION}

In inclusive hadron interactions at collider energies, $t \bar{t}$ pair production proceeds through partonic hard-scattering processes involving initial-state light quarks $q$ and gluons $g$. In lowest-order perturbative quantum chromodynamics (QCD), at $\mathcal{O}\left(\alpha_{s}^{2}\right)$, the two partonic subprocesses are $q+\bar{q} \rightarrow t+\bar{t}$ and $g+g \rightarrow t+\bar{t}$. Calculations of the cross section through next-to-leading order, $\mathcal{O}\left(\alpha_{s}^{3}\right)$, involve gluonic radiative corrections to these lowest-order subprocesses as well as contributions from the $q+g$ initial state [1]. In this paper, we describe calculations that go beyond fixed-order perturbation theory through resummation of the effects of gluon radiation [2-4] to all orders in the strong coupling strength $\alpha_{s}$.

The physical cross section is obtained through the factorization theorem

$$
\sigma_{i j}(S, m)=\frac{4 m^{2}}{S} \int_{0}^{\frac{S}{4 m^{2}}-1} d \eta \Phi_{i j}(\eta, \mu) \hat{\sigma}_{i j}(\eta, m, \mu) .
$$

The square of the total hadronic center-of-mass energy is $S$, the square of the partonic center-of-mass energy is $s, m$ denotes the top mass, $\mu$ is the usual

1) Paper presented by E. L. Berger, to be published in the Proceedings of DIS'97, Fifth International Workshop on Deep Inelastic Scattering and QCD, Chicago, IL, April 14 - 18, 1997. Argonne report ANL-HEP-CP-97-33. This work was supported by the US Department of Energy, Division of High Energy Physics, Contract No.W-31-109-ENG-38. 
factorization and renormalization scale, and $\Phi_{i j}(\eta, \mu)$ is the parton flux. The variable $\eta=\frac{s}{4 m^{2}}-1$ measures the distance from the partonic threshold. The indices ij $\in\{q \bar{q}, g g\}$ denote the initial parton channel. The partonic cross section $\hat{\sigma}_{i j}(\eta, m, \mu)$ is obtained either from fixed-order QCD calculations [1], or, as described here, from calculations that include of resummation [2-4] to all orders in $\alpha_{s}$. We use the notation $\alpha \equiv \alpha(\mu=m) \equiv \alpha_{s}(m) / \pi$. The total physical cross section is obtained after incoherent addition of the contributions from the the $q \bar{q}$ and $g g$ production channels.

Comparison of the partonic cross section at next-to-leading order with its lowest-order value reveals that the ratio becomes very large in the nearthreshold region. Indeed, as $\eta \rightarrow 0$, the " $K$-factor" at the partonic level $\hat{K}(\eta)$ grows in proportion to $\alpha \ln ^{2}(\eta)$. The very large mass of the top quark notwithstanding, the large ratio $\hat{K}(\eta)$ makes it evident that the next-to-leading order result does not necessarily provide a reliable quantitative prediction of the top quark production cross section at the energy of the Tevatron collider. Analogous examples include the production of hadronic jets that carry large values of transverse momentum and the production of pairs of supersymmetric particles with large mass.

\section{GLUON RADIATION AND RESUMMATION}

The origin of the large threshold enhancement may be traced to initialstate gluonic radiative corrections to the lowest-order channels. We remark that we are calculating the inclusive total cross section for the production of a top quark-antiquark pair, i.e., the total cross section for $t+\bar{t}+$ anything. The partonic subenergy threshold in question is the threshold for $t+\bar{t}+$ any number of gluons. This coincides with the threshold in the invariant mass of the $t+\bar{t}$ system for the lowest order subprocesses only.

For $i+j \rightarrow t+\bar{t}+g$, we define the variable $z$ through the invariant $(1-z)=\frac{2 k \cdot p_{t}}{m^{2}}$, where $k$ and $p_{t}$ are the four-vector momenta of the gluon and top quark. In the limit that $z \rightarrow 1$, the radiated gluon carries zero momentum. After cancellation of soft singularities and factorization of collinear singularities in $\mathcal{O}\left(\alpha_{s}^{3}\right)$, there is a left-over integrable large logarithmic contribution to the partonic cross section associated with initial-state gluon radiation. This contribution is often expressed in terms of "plus" distributions. In $\mathcal{O}\left(\alpha_{s}^{3}\right)$, it is proportional to $\alpha^{3} \ln ^{2}(1-z)$. When integrated over the near-threshold region $1 \geq z \geq 0$, it provides an excellent approximation to the full next-to-leading order physical cross section as a function of the top mass. The goal of gluon resummation is to sum the series in $\alpha^{n+2} \ln ^{2 n}(1-z)$ to all orders in $\alpha$ in order to obtain a more trustworthy prediction.

Different methods of resummation differ in theoretically and phenomenologically important respects. Formally, if not explicitly in some approaches, an integral over the radiated gluon momentum $z$ must be done over regions 
in which $z \rightarrow 1$. Therefore, one significant distinction among methods has to do with how the inevitable "non-perturbative" region is handled.

The method of resummation we employ [3] is based on a perturbative truncation of principal-value (PV) resummation [5]. This approach has an important technical advantage in that it does not depend on arbitrary infrared cutoffs. Because extra scales are absent, the method permits an evaluation of its perturbative regime of applicability, i.e., the region of the gluon radiation phase space where perturbation theory should be valid. We work in the $\overline{\mathrm{MS}}$ factorization scheme.

Factorization and evolution lead directly to exponentiation of the set of large threshold logarithms in moment $(n)$ space in terms of an exponent $E^{P V}$. The function $E^{P V}$ is finite, and $\lim _{n \rightarrow \infty} E^{P V}\left(n, m^{2}\right)=-\infty$. Therefore, the corresponding partonic cross section is finite as $z \rightarrow 1(n \rightarrow+\infty)$. The function $E^{P V}$ includes both perturbative and non-perturbative content. The non-perturbative content is not a prediction of perturbative QCD. We choose to use the exponent only in the interval in moment space in which the perturbative content dominates. We derive a perturbative asymptotic representation of $E(x, \alpha(m))$ that is valid in the moment-space interval

$$
1<x \equiv \ln n<t \equiv \frac{1}{2 \alpha b_{2}} .
$$

The coefficient $b_{2}=\left(11 C_{A}-2 n_{f}\right) / 12$; the number of flavors $n_{f}=5 ; C_{q \bar{q}}=$ $C_{F}=4 / 3 ;$ and $C_{g g}=C_{A}=3$.

The perturbative asymptotic representation is

$$
E_{i j}(x, \alpha) \simeq E_{i j}(x, \alpha, N(t))=2 C_{i j} \sum_{\rho=1}^{N(t)+1} \alpha^{\rho} \sum_{j=0}^{\rho+1} s_{j, \rho} x^{j}
$$

Here

$$
s_{j, \rho}=-b_{2}^{\rho-1}(-1)^{\rho+j} 2^{\rho} c_{\rho+1-j}(\rho-1) ! / j ! ;
$$

and $\Gamma(1+z)=\sum_{k=0}^{\infty} c_{k} z^{k}$, where $\Gamma$ is the Euler gamma function. The number of perturbative terms $N(t)$ in Eq. (3) is obtained [3] by optimizing the asymptotic approximation $|E(x, \alpha)-E(x, \alpha, N(t))|=$ minimum. Optimization works perfectly, with $N(t)=6$ at $m=175 \mathrm{GeV}$. As long as $n$ is in the interval of Eq. (2), all the members of the family in $n$ are optimized at the same $N(t)$, showing that the optimum number of perturbative terms is a function of $t$, i.e., of $m$ only.

Resummation is completed in a finite number of steps. Upon using the running of the coupling strength $\alpha$ up to two loops only, all monomials of the form $\alpha^{k} \ln ^{k+1} n, \alpha^{k} \ln ^{k} n$ are produced in the exponent of Eq. (3). We discard monomials $\alpha^{k} \ln ^{k} n$ in the exponent because of the restricted leading-logarithm 
universality between $t \bar{t}$ production and massive lepton-pair production, the Drell-Yan process.

The exponent we use is the truncation

$$
E_{i j}(x, \alpha, N)=2 C_{i j} \sum_{\rho=1}^{N(t)+1} \alpha^{\rho} s_{\rho} x^{\rho+1},
$$

with the coefficients $s_{\rho} \equiv s_{\rho+1, \rho}=b_{2}^{\rho-1} 2^{\rho} / \rho(\rho+1)$. This expression contains no factorially-growing (renormalon) terms. It is valuable to stress that we can derive the perturbative expressions, Eqs. (2), (3), and (4), without the principal-value prescription, although with less certitude [3].

After inversion of the Mellin transform from moment space to the physically relevant momentum space, the resummed partonic cross sections, including all large threshold corrections, can be written

$$
\hat{\sigma}_{i j}^{R ; p e r t}(\eta, m)=\int_{z_{\min }}^{z_{\max }} d z \mathrm{e}^{E_{i j}\left(\ln \left(\frac{1}{1-z}\right), \alpha\right)} \hat{\sigma}_{i j}^{\prime}(\eta, m, z) .
$$

The leading large threshold corrections are contained in the exponent $E_{i j}(x, \alpha)$, a calculable polynomial in $x$. The derivative $\hat{\sigma}_{i j}^{\prime}(\eta, m, z)=$

$d\left(\hat{\sigma}_{i j}^{(0)}(\eta, m, z)\right) / d z$, and $\hat{\sigma}_{i j}^{(0)}$ is the lowest-order $\mathcal{O}\left(\alpha_{s}^{2}\right)$ partonic cross section expressed in terms of inelastic kinematic variables. The lower limit of integration, $z_{\min }$, is fixed by kinematics. The upper limit, $z_{\max }<1$, is set by the boundary between the perturbative and non-perturbative regimes, well specified within the context of our calculation. Its presence assures us that our integration over the soft-gluon momentum is carried out only over a range in which poorly specified non-universal subleading terms would not contribute significantly even if retained. We cannot justify continuing the results of leading-logarithm resummation into the region $z>z_{\max }$.

Perturbative resummation probes the threshold down to $\eta \geq \eta_{0}=(1-$ $\left.z_{\max }\right) / 2$. Below this value, perturbation theory is not to be trusted. For $m$ $=175 \mathrm{GeV}$, we determine that the perturbative regime is restricted to values of the subenergy greater than $1.22 \mathrm{GeV}$ above the threshold $(2 m)$ in the $q \bar{q}$ channel and $8.64 \mathrm{GeV}$ above threshold in the $g g$ channel. The difference reflects the larger color factor in the $g g$ case. The value $1.22 \mathrm{GeV}$ is comparable to the decay width of the top quark, a natural definition of the perturbative boundary and by no means unphysically large.

\section{PHYSICAL CROSS SECTION}

Other than the top mass, the only undetermined scales are the QCD factorization and renormalization scales. We adopt a common value $\mu$ for both. In Fig. 1, we show our total cross section for $t \bar{t}$-production as a function of top mass in $p \bar{p}$ collisions at $\sqrt{S}=1.8 \mathrm{TeV}$. The central value is obtained 
with the choice $\mu / m=1$, and the lower and upper limits are the maximum and minimum of the cross section in the range $\mu / m \in\{0.5,2\}$. At $m=175$ $\mathrm{GeV}$, the full width of the uncertainty band is about $10 \%$. As is to be expected, less variation with $\mu$ is evident in the resummed cross section than in the next-to-leading order cross section. In estimating uncertainties, we do not consider explicit variations of our non-perturbative boundary, expressed through $z_{\max }$. This is justified because, for a fixed $m$ and $\mu, z_{\max }$ is obtained by enforcing dominance of the universal leading logarithmic terms over the subleading ones. Therefore, $z_{\max }$ is derived and is not a source of uncertainty. At fixed $m$, the boundary necessarily varies as $\mu$ and thus $\alpha$ vary.

Our calculation is in agreement with the data [6]. We find $\sigma^{t \bar{t}}(m=$ $175 \mathrm{GeV}, \sqrt{S}=1.8 \mathrm{TeV})=5.52_{-0.42}^{+0.07} \mathrm{pb}$. This cross section is larger than the next-to-leading order value by about $9 \%$. The top quark cross section increases quickly with the energy of the $p \bar{p}$ collider. We determine $\sigma^{t \bar{t}}(m=175 \mathrm{GeV}, \sqrt{S}=2 \mathrm{TeV})=7.56_{-0.55}^{+0.10} \mathrm{pb}$. The central value rises to $22.4 \mathrm{pb}$ at $\sqrt{S}=3 \mathrm{TeV}$ and $46 \mathrm{pb}$ at $\sqrt{S}=4 \mathrm{TeV}$.

Extending our calculation to much larger values of $m$ at $\sqrt{S}=1.8 \mathrm{TeV}$, we find that resummation in the principal $q \bar{q}$ channel produces enhancements over the next-to-leading order cross section of $21 \%, 26 \%$, and $34 \%$, respectively, for $m=500,600$, and $700 \mathrm{GeV}$. The reason for the increase of the enhancements with mass at fixed energy is that the threshold region becomes increasingly dominant. Since the $q \bar{q}$ channel also dominates in the production of hadronic jets at very large values of transverse momenta, we suggest that on the order of $20 \%$ of the excess cross section reported by the CDF collaboration [7] may

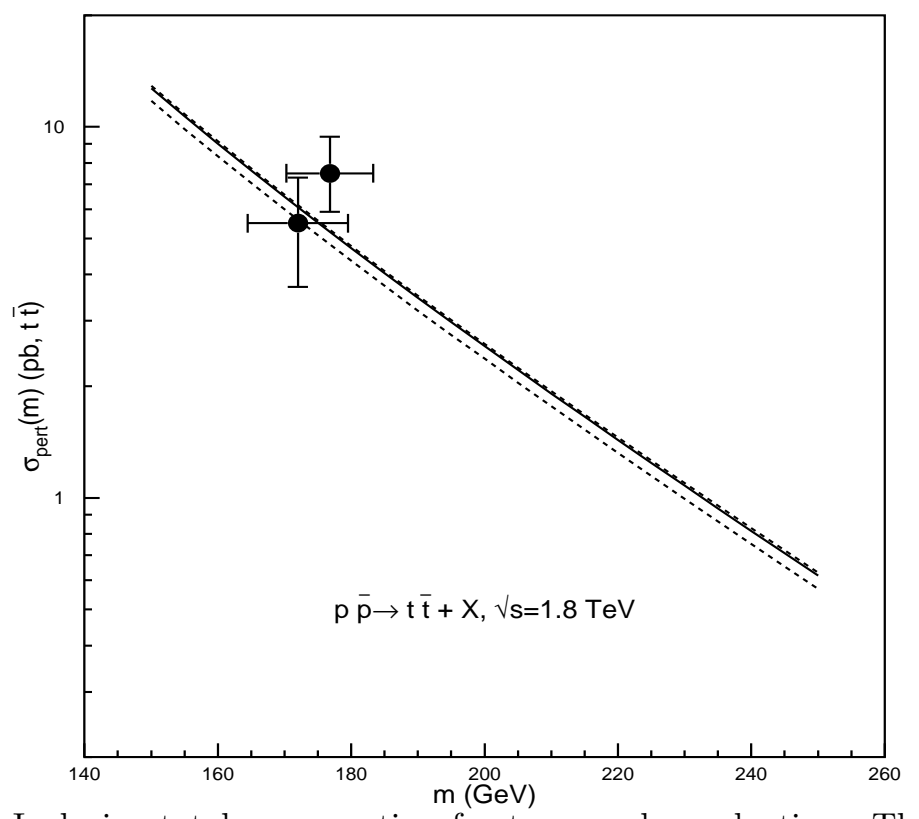

FIGURE 1. Inclusive total cross section for top quark production. The dashed curves show the upper and lower limits while the solid curve is our central prediction. CDF and D0 data are shown. 
be accounted for by resummation.

\section{OTHER METHODS OF RESUMMATION}

Two other groups have published calculations of the total cross section at $m=175 \mathrm{GeV}$ and $\sqrt{s}=1.8 \mathrm{TeV}: \sigma^{t \bar{t}}(\operatorname{LSvN}[2])=4.95_{-0.40}^{+0.70} \mathrm{pb}$; and $\sigma^{t \bar{t}}(\mathrm{CMNT}[4])=4.75_{-0.68}^{+0.63} \mathrm{pb}$. From a numerical point of view, all agree within their estimates of theoretical uncertainty. However, the resummation methods differ as do the methods for estimating uncertainties. Both the central value and the band of uncertainty of the LSvN predictions are sensitive to their infrared cutoffs. To estimate theoretical uncertainty, we use the standard $\mu$-variation, whereas LSvN obtain theirs primarily from variations of their cutoffs. It is difficult to be certain of the central value and to evaluate theoretical uncertainties in a method that requires an undetermined infrared cutoff.

The group of Catani, Mangano, Nason, and Trentadue (CMNT) [4] calculate a central value of the resummed cross section (also with $\mu / m=1$ ) that is less than $1 \%$ above the exact next-to-leading order value. There are similarities and differences between our approach and the method of CMNT. We use the same universal leading-logarithm expression in moment space, but differences occur after the transformation to momentum space. The differences can be stated more explicitly if we examine the perturbative expansion of the resummed hard kernel $\mathcal{H}_{i j}^{R}(z, \alpha)$. If, instead of restricting the resummation to the universal leading logarithms only, we were to use the full content of $\mathcal{H}_{i j}^{R}(z, \alpha)$, we would arrive at an analytic expression that is equivalent to the numerical inversion of Ref. [4],

$$
\mathcal{H}_{i j}^{R} \simeq 1+2 \alpha C_{i j}\left[\ln ^{2}(1-z)+2 \gamma_{E} \ln (1-z)\right]+\mathcal{O}\left(\alpha^{2}\right)
$$

In terms of this expansion, in our work we retain only the leading term $\ln ^{2}(1-$ $z$ ) at order $\alpha$, but both this term and the non-universal subleading term $2 \gamma_{E} \ln (1-z)$ are retained in Ref. [4]. If this subleading term is discarded in Eq. (7), the residuals $\delta_{i j} / \sigma_{i j}^{N L O}$ defined in Ref. [4] increase from $0.18 \%$ to $1.3 \%$ in the $q \bar{q}$ production channel and from $5.4 \%$ to $20.2 \%$ in the $g g$ channel. After addition of the two channels, the total residual $\delta / \sigma^{N L O}$ grows from the negligible value of about $0.8 \%$ to the value $3.5 \%$. While still smaller than the increase of $9 \%$ that we obtain, the increase of $3.5 \%$ vs. $0.8 \%$ shows the substantial influence of the subleading logarithmic terms retained in Ref. [4].

We judge that it is preferable to integrate over only the region of phase space in which the subleading term is suppressed numerically. Our reasons include the fact that the subleading term is not universal, is not the same as the subleading term in the exact $\mathcal{O}\left(\alpha^{3}\right)$ calculation and can be changed if one elects to keep non-leading terms in moment space. The subleading term is negative and numerically very significant when it is integrated throughout 
phase space (i.e., into the region of $z$ above our $z_{\max }$ ). In the $q \bar{q}$ channel at $m=175 \mathrm{GeV}$ and $\sqrt{S}=1.8 \mathrm{TeV}$, its inclusion eliminates more than half of the contribution from the leading term. Although the goal is to resum the threshold contributions responsible for the enhancement of the cross section at next-to-leading order, the method of Ref. [4] does not reproduce most of this enhancement. The influence of non-universal subleading terms is amplified at higher orders where additional subleading structures occur in the approach of Ref. [4] with significant numerical coefficients proportional to $\pi^{2}, \zeta(3)$, and so forth. In our view, the results of a leading-logarithm resummation should not rely on subleading structures in any significant manner. The essence of our determination of the perturbative boundary $z_{\max }$ is precisely that below $z_{\max }$ subleading structures are also numerically subleading, whether or not classes of poorly substantiated subleading logarithms are included. For a more detailed discussion of these and other points of difference, including issues of momentum conservation and factorial growth, see section VI of Ref. [8].

Since the large threshold logarithms are mastered by resummation, the theoretical reliability of the resummed result is greater than that of a fixed-oder calculation. Our analysis and the stability of our cross section under variation of the hard scale $\mu$ provide confidence that our perturbative resummation yields an accurate calculation of the inclusive top quark cross section at Tevatron energies.

\section{REFERENCES}

1. Nason P., Dawson S., and Ellis, R. K., Nucl. Phys. B303, 607 (1988); B327, 49 (1989); B335, 260(E) (1990). Beenakker W. et al., Phys. Rev. D40, 54 (1989); Nucl. Phys. B351, 507 (1991).

2. Laenen E., Smith J., and van Neerven, W. L., Nucl. Phys. B369, 543 (1992); Phys. Lett. B 321, 254 (1994).

3. Berger, E. L., and Contopanagos H., Phys. Lett. B 361, 115 (1995); Phys. Rev. D54, 3085 (1996).

4. Catani S., Mangano M., Nason P., and Trentadue L., Phys. Lett. B 378, 329 (1996); Nucl. Phys. B478, 273 (1996); Mangano M., and Nason P., private communication.

5. Contopanagos H., and Sterman G., Nucl. Phys. B400, 211 (1993); B419, 77 (1994).

6. Tipton P., Proceedings of the 28th International Conference on High Energy Physics, Warsaw, July, 1996; ed. by Ajduk Z., and Wroblewski A. K., Singapore: World Scientific, 1997, pp. 123-131; Castro A. (CDF collaboration), ibid, pp. 851-854; S. Abachi (D0 Collaboration), hep-ex/9703008 and hep-ex/9704015.

7. Abe F. et al, Phys. Rev. Lett. 77, 438 (1996).

8. Berger E. L., and Contopanagos H., Argonne report ANL-HEP-PR-97-01 (hepph/9706206), May, 1997. 